\title{
Mechanism of TRPA1 and TRPV4 Participating in Mechanical Hyperalgesia of Rat Experimental Knee Osteoarthritis
}

\author{
Runlin XING, Peimin WANG, Linrui ZHAO, Bo XU, Nongshan ZHANG, Xiaochen LI \\ Department of Orthopedic, Affiliated Hospital of Nanjing University of Chinese Medicine, Nanjing, China
}

\begin{abstract}
Objectives: This study aims to observe both transient receptor potential ankyrin 1 (TRPA1) and transient receptor potential vanilloid 4 (TRPV4) expressions in synovial tissues of rats with mechanical hyperalgesia induced by experimental knee osteoarthritis (KOA).

Patients and methods: Forty-five four-month-old Sprague Dawley male rats, weight ranging from $440 \mathrm{~g}$ to $470 \mathrm{~g}$, were randomly allocated into three groups, namely KOA group, KOA-antagonist group, and normal group. Mechanical withdrawal thresholds of five rats from each group were detected one week before modeling, and two, four, six, and eight weeks after modeling, respectively. Synovial and cartilage tissues from diseased knee were collected after sacrificing the rats eight weeks after modeling so to observe pathological morphology at cartilage tissues and to determine protein and gene expressions of TRPA1 and TRPV4 at synovial tissues.

Results: Rats with KOA showed obvious mechanical hyperalgesia from two weeks after modeling to the latest follow-up, eight weeks after modeling The abnormally low level of mechanical withdrawal thresholds can be increased by TRPA1 and TRPV4 ion channel blockers.

Conclusion: Up-regulating expressions of TRPA1 and TRPV4 participate in the occurrence mechanism of mechanical hyperalgesia induced by KOA. Keywords: Knee osteoarthritis; mechanical hyperalgesia; occurrence mechanism; transient receptor potential ion channel.
\end{abstract}

Knee osteoarthritis (KOA) is a degenerative disease of bones and joints, commonly seen in middle aged and elderly people. ${ }^{1}$ Pain appears to be the most frequent manifestation, accounting $65 \%$ of KOA patients in clinic. ${ }^{2}$ Current studies regarding KOA pain mechanism mostly focus on the following three aspects: (i) synovial inflammation: pro-inflammatory cytokines such as histamine, prostaglandins, leukotriene, neuropeptides may activate nociceptor to induce pain; (ii) ostealgia: subchondral bone and marrow vessels and nerves exposure caused by KOA articular surface cartilage denudation may also lead to pain; (iii) decreased pain threshold: chronic pain in KOA demonstrates a decreased pain threshold, which can give rise to an increased pain intensity by pain stimulation in the same intensity (hyperalgesia) and even by non-painful stimulation (allodynia). ${ }^{3}$ Hyperalgesia is the primary cause for decreased pain threshold in chronic pain, which has been given more and more attention recently. $\mathrm{A}$ complete link of hyperalgesia includes the activation of peripheral nerve pain receptors, central nerves' integration, and the processing of pain signals. In light of varied activation conditions for peripheral nociceptors, hyperalgesia can be classified into two as mechanical and thermal (cold and warm). Previous studies have demonstrated the existence of peripheral hyperalgesia in KOA diseased region, and discovered manifestations of both cold and mechanical hyperalgesias in KOA pain, whose mechanism requires further study. ${ }^{5}$ Clinical studies have observed KOA pain and discovered its typical mechanical hyperalgesia feature, namely, severe persistent pain induced by minor activity. ${ }^{6}$ Thus, it is inferred that mechanical hyperalgesia

Received: May 03, 2016 Accepted: July 18, 2016 Published online: January 18, 2017

Correspondence: Peimin Wang, MD. Department of Orthopedic, Affiliated Hospital of Nanjing University of Chinese Medicine, 86210000 Nanjing, China.

Tel: 8615895977090 e-mail: drwpm@163.com

(02017 Turkish League Against Rheumatism. All rights reserved. 
may play a very significant role in $\mathrm{KOA}$ pain. If its mechanism for effective intervention were to be clarified and uncovered, it would provide a creative idea and innovating approach to control KOA pain.

Transient receptor potential (TRP) is a nonselective cation channel lying on cytomembranes and appears to have high permeability to calcium. ${ }^{7}$ Increased intracellular calcium relies on extracellular calcium influxes and intracellular calcium mobilization, in which TRP ion channel can both regulate. It is not only a voltage-gated or dependent calcium channel for excitable cells (nerve and muscle), but also a non-voltage-gated or dependent calcium channel for non-excitable cells (epithelial, endothelial, mucosa, bone, cartilage, and synovial membrane), and is able to convert and integrate varied thermal, mechanical, and chemical stimulations into electrical signals for transduction, respond to multiple cell and body thermal, touching and osmotic pressure stimulations so as to cause senses of touching, hearing and pain, thus making it the frontline guard of the sensory system and also a mutual ion channel mediating thermal and mechanical hyperalgesias. ${ }^{8}$ Hence, proteins mediating mechanical hyperalgesia in TRP ion channel have increasingly attracted the concern of academics. Transient receptor potential vanilloid 4 (TRPV4) and transient receptor potential ankyrin 1 (TRPA1) have already been main focuses for comparatively mature studies currently. Calcium in TRPV4 demonstrates moderate permeability and can be activated by various physical stimulations such as low permeability, touching pressure and shear forces. Previous studies have showed that TRPV4 is not only widely distributed in peripheral and central nerve system, but can also be transferred freely to peripheral nerve endings. ${ }^{9,10}$ TRPV4 knockout mice demonstrated abnormal reactions such as decreased sensitivity to osmotic pressure, hearing loss, and heat activation thermal threshold change, suggesting that TRPV4 plays a key role in physical (mechanical and thermal) factors mediated senses. ${ }^{11}$ TRPA1 can be activated by nociceptive cold stimulations lower than $17{ }^{\circ} \mathrm{C}$ as well as non-nociceptive mechanical stimulations. ${ }^{12}$ Its participation in inflammatory reaction as a synergist has also been proved, making it a significant participant inducing inflammatory pain. ${ }^{13}$
Meanwhile, we noticed some phenomenon relating to KOA pain. For one thing, inflammatory environment can affect TRP ion channel's mechanical sensitivity. For instance, low permeability can lead to cellular swelling and produce 5,6-Epoxyeicosatrienoic acid (5,6-EET) to directly activate TRP protein super-family members' sensitivity to mechanical factors such as TRPV4 and TRPA1. Previous studies have witnessed that prostaglandin E2 can significantly strengthen this process, which may explain nonsteroidal anti-inflammatory drugs' effect in relieving KOA pain to some extent. ${ }^{14}$ For another, there exists very close co-expressions and synergy effects among varied TRP ion channel family members. For instance, TRPV4 has a high homology of $40 \%$ with TRPV1; transient receptor potential melastatin 8 shares a similar signal pathway mechanism with TRPV1, which may explain KOA pain's double attributes of cold and warm hyperalgesias. ${ }^{15}$

Apart from its focus on nerve damaging pain previously, TRP ion channel has arisen general concern in light of its close relationship with chronic joint pain recently. Firstly, TRPV1 showed high expression in fibroblasts of KOA synovial joints. ${ }^{16}$ Later, relationships between expressions, open levels of TRPA1, transient receptor potential melastatin 8 and TRPV4, and pain in KOA rat models have also been verified. ${ }^{17}$ Since TRPA1 and TRPV4 are the two key participants in mechanical hyperalgesia, together with their high co-expression and synergy effect with TRPV1, it is concluded that TRPA1 and TRPV4 mediated mechanical hyperalgesia is more critical in KOA pain. Therefore, verifying the occurrence mechanism of mechanical hyperalgesia and coming up with targeted intervention will be of great significance for KOA treatment, especially in terms of pain relieving. Hence, in this study, we aimed to observe both TRPA1 and TRPV4 expressions in synovial tissues of rats with mechanical hyperalgesia induced by experimental KOA.

\section{PATIENTS AND METHODS}

This study was conducted at Affiliated Hospital Of Nanjing University Of Chinese Medicine, Department of Orthopedic Hospital between 
June 1, 2015 and June 1, 2016. In the study, we used 45 four-month-old Sprague Dawley male rats, weight ranging from $440 \mathrm{~g}$ to $470 \mathrm{~g}$ (provided by Beijing Vital River Laboratory Animal Technology Co. Ltd.); Von Frey Hairs analgesia meter; Anti-TRPA1 antibody (ab58844; provided by American Abcam Co.); Anti-TRPV4 antibody (ab94868; provided by American Abcam Co.); Selective TRPA1 ion channel blocker, TCS 5861528 (provided by American Alpha Applied Bioscience Co.); Selective TRPV4 ion channel blocker, GSK 2193874 (provided by American Alpha Applied Bioscience Co.). The study protocol was approved by the Affiliated Hospital of Nanjing University of Chinese Medicine, Department of Orthopedic Hospital Ethics Committee. The study was conducted in accordance with the principles of the Declaration of Helsinki.

Rats were randomly allocated into three groups, including the KOA group, KOA-antagonist group (applied selective TRPA1 and TRPV4 channel blockers two weeks after modeling orally; TCS 5861528 + GSK 2193874: $0.3 \mathrm{mg} / \mathrm{kg}$ two days), and normal group in accordance with a random number generated by SPSS version 11.0 software (SPSS Inc., Chicago, IL, USA).

Preoperative fasting for 12 hours and weighting were performed. Rats were anesthetized via intraperitoneal injection of $3 \%$ sodium pentobarbital $(30 \mathrm{mg} / \mathrm{kg})$ and fixed in the supine position to shave both knee joints. Routine disinfection was performed at operation scope. In aseptic condition, subcutaneous tissue and joint capsule was cut open along knee joint anterior midline for dislocating lateral patella by overextending knee joints (normally toward lateral knee direction). Anterior cruciate ligament was cut off with ophthalmic scissors after bending knee joints. Suture was performed layer by layer to close the wound after stretching out knee joints to reset the patella. Later, anterior drawer test was conducted to determine operation results. Rats were given amikacin $(10 \mathrm{mg} / \mathrm{kg})$ for four days to prevent inflammation, and kept in separate cages, with free access to forage and drinking water, free activities and without joint fixation. Animal room temperature was kept at $25 \pm 2{ }^{\circ} \mathrm{C}$, with a relative humidity of $60 \pm 5 \%$ and lighting for 12 hours.

Before modeling, rats were given adaptive tests for several days; their normal thresholds for mechanical stimulation induced pain were obtained to work as the basic threshold of the experiment. Von Frey filaments were applied as mechanical stimulation for pain threshold determination and no movements such as seeking, defecation, urination or perching on the hind legs were observed before pain threshold determination. Metal probe was used to stimulate vertically rats' left and right feet soles. Stimulation intensity was gradually increased to induce the rats' rapid paw withdrawal, lifting, and licking reactions in three seconds. Recorder automatically wrote down the minimal stimulating intensity needed to induce paw withdrawal, and this stimulation action would be performed again and again (every 1 minute) until the recorder had three similar test results, whose average value would work as the mechanical withdrawal threshold (MWT). Both left and right leg MWTs of all rats were detected preoperatively, and at postoperative second, fourth, sixth, and eighth weeks at two fixed time points, namely 8 am and $2 \mathrm{pm}$. All rats were habituated and acclimated to testing environment 30 minutes before test.

Cartilages were collected after sacrificing the rats, then fixed in 10\% neutral formalin, soaked in ethylenediaminetetraacetic acid, embedded in paraffin, and cut into slices for routine hematoxylin and eosin staining. Later, stained cartilage slices of each group were evaluated and given scores in accordance with the cartilage hematoxylin and eosin staining evaluation standard recommended by the Osteoarthritis Research Society International (Table 1). ${ }^{18}$

Synovial tissues were collected after sacrificing the rats, and then fixed in 10\% neutral formalin, soakedinethylenediaminetetraaceticacid, embedded in paraffin and cut into slices, then subjected to conventional dewaxing hydration, incubated in $0.5 \%$ hydrogen peroxide solution for 10 minutes and then washed away with water. Afterwards, slices were immersed in ethylenediaminetetraacetic acid, heated in the stainless steel pressure cooker for 1-2 minutes after the cooker reached exhaust temperature and pressure. Slices were then taken out of the cooker and put into phosphate-buffered saline, incubated with the appropriate dilution concentrated primary antibody, overnight at $4{ }^{\circ} \mathrm{C}$, washed with phosphate-buffered saline, incubated with secondary antibody for 20 minutes and again washed with phosphate-buffered saline. Each 
Table 1. Mankin score for histological evaluation

\begin{tabular}{|c|c|c|c|c|}
\hline Score & Structure staining & Chondrocyte loss & Safranin O & Tidemark integrity \\
\hline 0 & Normal & No decrease in cells & $\begin{array}{l}\text { Uniform staining throughout } \\
\text { articular cartilage }\end{array}$ & Intact \\
\hline 1 & Surface irregularities & Minimal decrease in cells & $\begin{array}{l}\text { Loss of staining in the } \\
\text { superficial zone for less than } \\
\text { one-half of the length of the } \\
\text { condyle or plateau }\end{array}$ & Crossed by blood vessels \\
\hline 2 & $>3$ superficial clefts & Moderate decrease in cells & $\begin{array}{l}\text { Loss of staining in the } \\
\text { superficial zone for one-half } \\
\text { or more of the length of the } \\
\text { condyle or plateau }\end{array}$ & \\
\hline 3 & 1-3 superficial clefts & Marked decrease in cells & $\begin{array}{l}\text { Loss of staining in the } \\
\text { superficial and middle zones } \\
\text { for less than one-half of the } \\
\text { length of the condyle or } \\
\text { plateau }\end{array}$ & \\
\hline 4 & $\begin{array}{l}\text { 1-3 clefts extending into the } \\
\text { middle zone }\end{array}$ & $\begin{array}{c}\text { Very extensive decrease in } \\
\text { cells }\end{array}$ & $\begin{array}{l}\text { Loss of staining in the } \\
\text { superficial and middle zones } \\
\text { for one-half or more of the } \\
\text { length of the condyle or } \\
\text { plateau }\end{array}$ & \\
\hline 5 & $\begin{array}{l}\text { 1-3 clefts extending into the } \\
\text { deep zone }\end{array}$ & & $\begin{array}{l}\text { Loss of staining in all three } \\
\text { zones for less than one-half } \\
\text { of the length of the condyle } \\
\text { or plateau }\end{array}$ & \\
\hline 6 & $\begin{array}{l}\text { Clefts extending to calcified } \\
\text { cartilage }\end{array}$ & & $\begin{array}{l}\text { Loss of staining in all three } \\
\text { zones for one-half or more of } \\
\text { the length of the condyle or } \\
\text { plateau }\end{array}$ & \\
\hline
\end{tabular}

slice was then stained with 3,3'-diaminobenzidine staining, restained with hematoxylin and eosin dehydrated with graded alcohol and mounted using neutral gum.

Total protein extraction: Synovium tissues were weighed mixed with RIPA lysate (Shanghai Beyotime Biotechnology Co., Ltd.) and grinded for 10-15 minutes. Samples were agitated on ice for 30 minutes to remove the supernatant and the protein levels were quantified with a bicinchoninic acid protein assay kit.

Detailed procedures of Western-blot: The protein samples were electrophoresed in sodium dodecyl sulfate polyacrylamide gel electrophoresis to separate protein bands. Proteins were transferred from gel onto polyvinylidene difluoride membrane, blocked with 5\% non-fat dry milk for two hours. The membrane was incubated with first antibody, overnight at $4{ }^{\circ} \mathrm{C}$. On the next day, membrane was incubated with second antibody for two hours. Later, bands were visualized by exposure to enhanced chemiluminescence method and the overall gray value of protein bands (average gray value $\times$ gray value area) was quantified, $\beta$-actin as internal marker, namely, target protein gray value/ internal reference overall gray value.

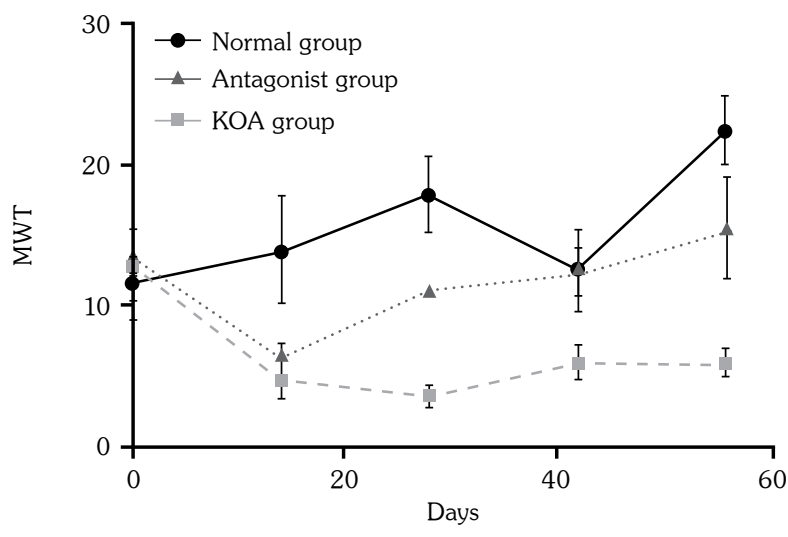

Figure 1. Mechanical withdrawal thresholds of rats in normal, knee osteoarthritis, and knee osteoarthritisantagonist groups. MWT: Mechanical withdrawal threshold; KOA: Knee osteoarthritis. 


\begin{tabular}{|c|c|c|c|c|c|}
\hline \multirow[t]{2}{*}{ Group (n=15) } & Preoperative & $14^{\text {th }}$ day & $28^{\text {th }}$ day & $42^{\text {nd }}$ day & $56^{\text {th }}$ day \\
\hline & Mean \pm SEM & Mean \pm SEM & Mean \pm SEM & Mean \pm SEM & Mean \pm SEM \\
\hline Normal & $12.667 \pm 7.090$ & $14.000 \pm 9.295$ & $17.833 \pm 6.616$ & $12.500 \pm 7.148$ & $22.333 \pm 5.680$ \\
\hline Knee osteoarthritis & $13.167 \pm 6.706$ & $4.667 \pm 2.733^{* * *}$ & $3.667 \pm 1.506^{* *}$ & $6.000 \pm 2.828^{* *}$ & $6.000 \pm 2.191^{* *}$ \\
\hline Antagonist & $13.667 \pm 6.831$ & $6.333 \pm 2.338 \Delta$ & $11.167 \pm 4.446 \Delta \Delta$ & $12.333 \pm 4.179 \Delta \Delta$ & $15.500 \pm 8.526 \Delta \Delta$ \\
\hline
\end{tabular}

Total ribonucleic acid (RNA) extraction: RNA was isolated from synovium with trizol. RNA concentration and purity were measured by spectrophotometer, A260 nm/A280 nm ratio being 1.8 2.0.

Reverse transcription reaction: Total RNA from each group and $10 \mathrm{uL}$ polymerase chain reaction (PCR) solution were added into Prime Script RT reagent Kit $10 \mathrm{uL}$ for reverse transcription at condition of $37{ }^{\circ} \mathrm{C}$ (15 minutes) and $85{ }^{\circ} \mathrm{C}$ ( 5 seconds) and later kept at $4{ }^{\circ} \mathrm{C}$.

Primer design: Primer was designed and synthesized by Shanghai Biotechnology Service Company in accordance with Gene sequence in GenBank Gene sequence design, together with Oligo v6.6. Sequences for TRPA1, TRPV4 as well as internal reference gene GAPDH were as follows. Sequences of primers used for TRPA1: 5'-GCAGCATTTTCAGGTGCCAA-3' (forward), primer length: 172 bp 5'CGCTGTCCAGGCACATCTTA-3' (reverse), primer length: $172 \mathrm{bp}$. Sequences of primers used for TRPV4: 5'-CTTTACTTCACCCGTGGGCT-3' (forward), primer length: 188 bp,
5' - C A G T T G C T C T G G T C C T C G T T- 3 ' (reverse), primer length: 188 bp. Sequences of primers used for GAPDH: 5'-ACAGCAACAGGGTGGGTGGTGGAC-3' (forward), primer length: 167 bp, 5'-TTTGAGGGTGCAGCGAACTT-3' (reverse), primer length: $167 \mathrm{bp}$. Fluorogenic quantitative PCR: 2 uL complementary deoxyribonucleic acid was added into $8 \mathrm{uL}$ RNase-free water, $8 \mathrm{uL} 2.5$ RealMasterMix, 1 uL 20 SYBR Green, with a primer pair of $0.5 / 0.5 \mathrm{uL}$. Reaction condition includes one cycle of predegeneration at $94{ }^{\circ} \mathrm{C}$ for four minutes, and forty cycles of degeneration at $94{ }^{\circ} \mathrm{C}$ for 30 seconds, annealing at $60{ }^{\circ} \mathrm{C}$ for 30 seconds, extending at $72{ }^{\circ} \mathrm{C}$ for 20 seconds. The above PCR product was held at $95{ }^{\circ} \mathrm{C}$ for one minute, cooled into $55{ }^{\circ} \mathrm{C}$, and then heated up between $55-95{ }^{\circ} \mathrm{C}, 0.2{ }^{\circ} \mathrm{C} /$ second before drawing solubility curve. Later, $2^{-{ }^{\Delta} \mathrm{CT}}$ calculation $\left({ }^{\wedge} \mathrm{CT}=\right.$ Sample cycle threshold valueInternal reference cycle threshold value) was used to analyze the relative expression of target genes, GAPDH as internal reference gene for semiquantitative calculation.
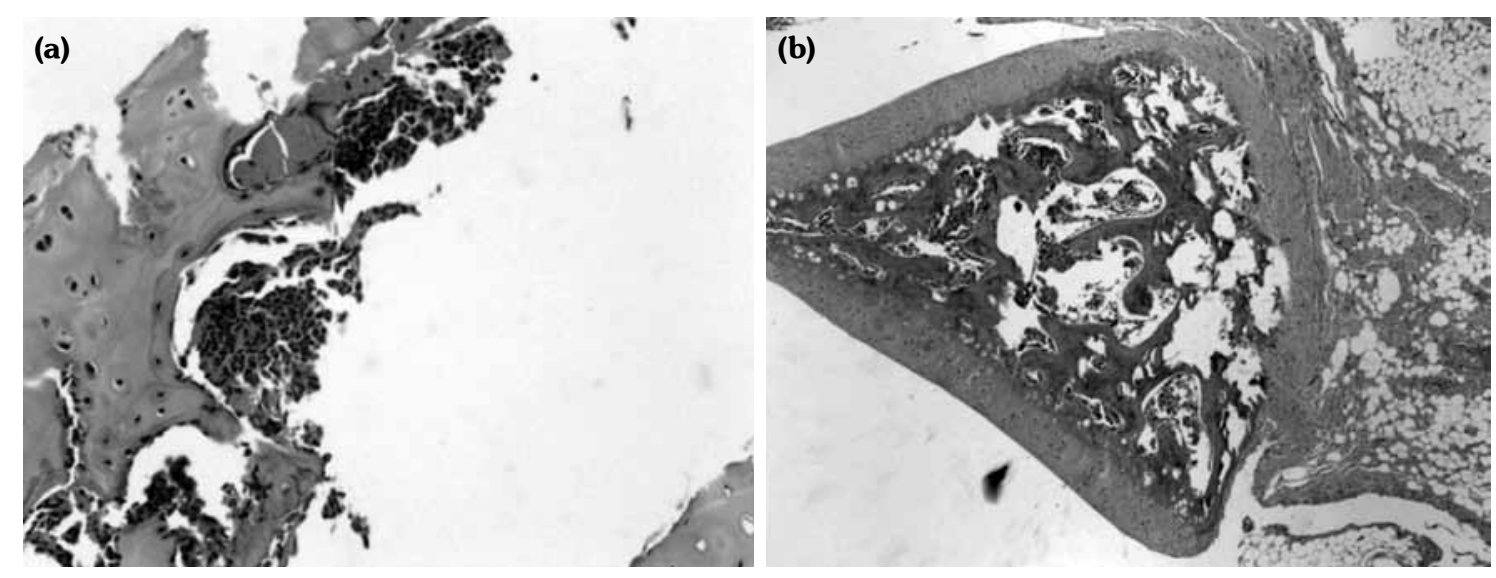

Figure 2. (a) Cartilage histomorphology of osteoarthritis knee joint $(H-E \times 200)$. (b) Cartilage histomorphology of healthy knee joint (safranin O staining, $\times 200$ ). 
Table 3. Histological assessment of cartilage of normal and knee osteoarthritis groups

\begin{tabular}{lcr}
\hline Group & & Mankin score \\
\cline { 2 - 3 } Normal & $\mathrm{n}$ & Mean \pm SEM \\
Knee osteoarthritis & 15 & $4.7 \pm 0.95^{*}$ \\
SEM: Standard error of the mean; * $\mathrm{p}<0.05 ;{ }^{* *} \mathrm{p}<0.01$, compared with \\
normal group.
\end{tabular}

\section{Statistical analysis}

All results were expressed as mean \pm standard deviation. Threshold data were analyzed using a two-way analysis of variances (Two-way ANOVA and Dunnett's test, SPSS 11.0), one-way ANOVA for group differences comparison, least significant difference test for group comparison with variance homogeneity, and Tamhane's $T_{2}$ test for group comparison with variance heterogeneity. $\mathrm{P}<0.05$ was considered as statistically different, while $p<0.01$ was considered as significantly different.

\section{RESULTS}

Mechanical withdrawal thresholds of KOA group were significantly lower than those in normal group and the condition sustained until the last observation conducted eight weeks after modeling. The usages of TRPA1 and TRPV4 inhibitors since two weeks after modeling obviously extended MWTs in KOA-antagonist group, but still MWTs were shorter then those in normal group (Figure 1, Table 2).

In KOA group, joint cartilage demonstrated rough and irregular surface, yellow with loss of normal luster and elasticity and had distinct bunchy fiber changes, fracturing, eroded changes, softening and coloboma. In normal group, joint cartilage remained semitransparent with luster and elasticity, smooth edge, and showed no fracturing, softening, defects or osteophyte formation (Figure 2).

Light microscopic observation and Mankin's evaluation revealed defects on cartilage surface, full-thickness chondral defects with many
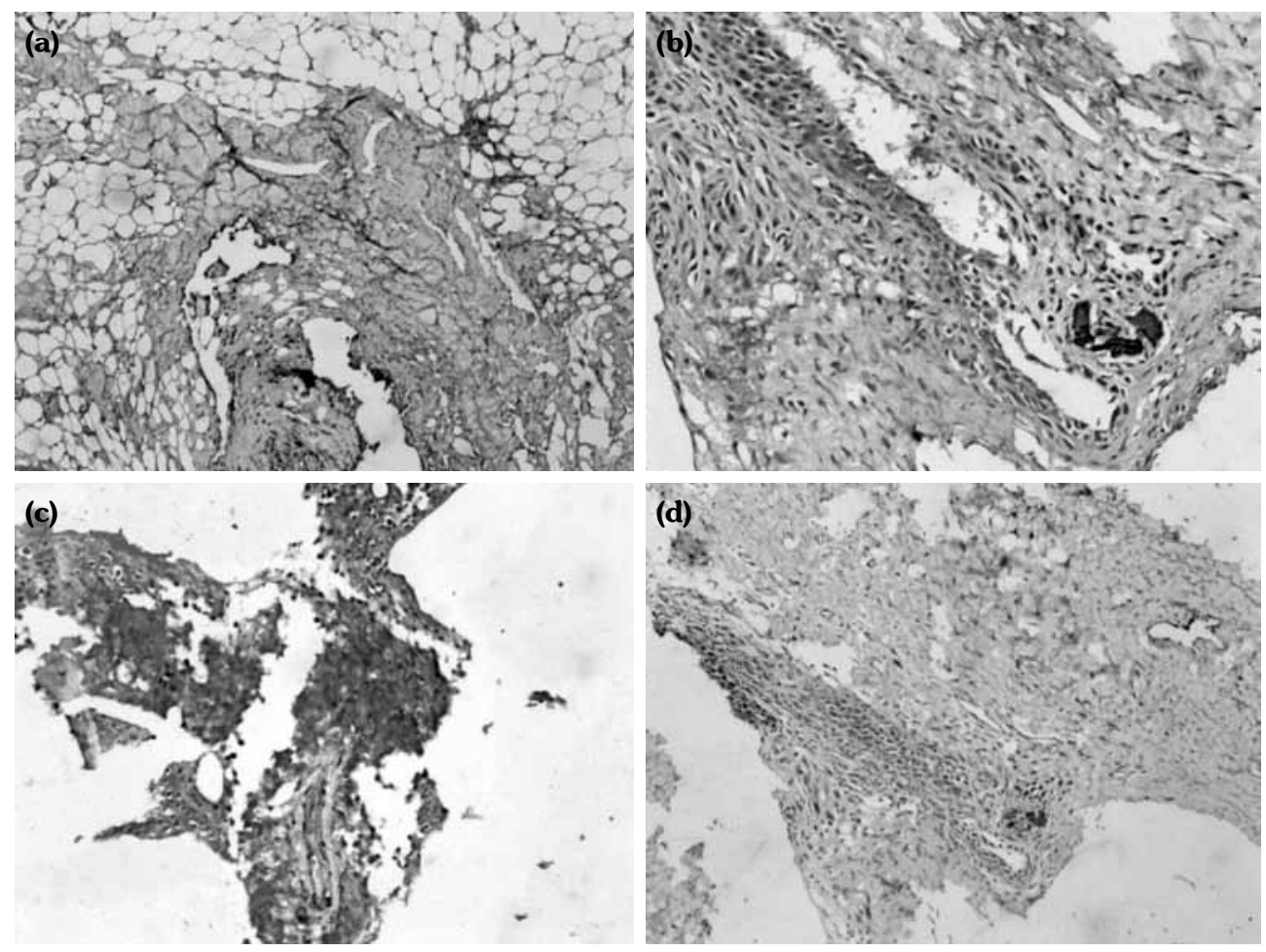

Figure 3. (a, b) Transient receptor potential ankyrin 1 in synovial tissues of normal and knee osteoarthritis groups detected by indirect immunofluorescence. (c, d) Transient receptor potential vanilloid 4 in synovial tissues of normal and knee osteoarthritis groups detected by indirect immunofluorescence (immunohistochemistry, 3, 3'-diaminobenzidine (DAB) as organic reagent, hematoxylin counterstain, $\times 400$ ). 

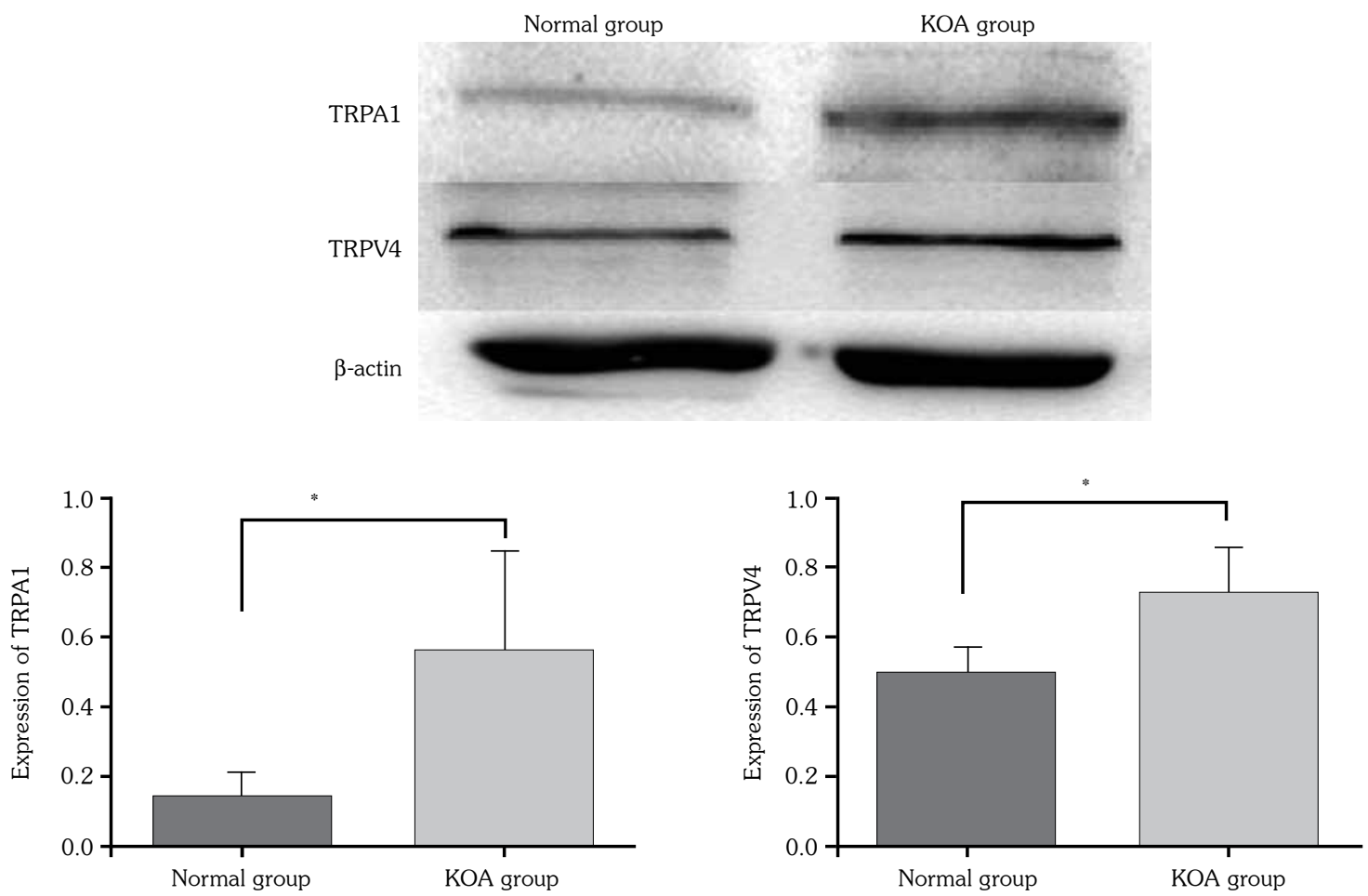

Figure 4. Protein expression of transient receptor potential ankyrin 1 and transient receptor potential vanilloid 4 in synovium tissue of knee joint. TRPA1: Transient receptor potential ankyrin 1; TRPV4: Transient receptor potential vanilloid 4; KOA: Knee osteoarthritis.

fissurations downward extending to radiating layer, blurring in layering and irregular in structure, tidemark broken and calcified layer distinctly thickened in KOA group. In normal group, cartilage surface appeared smooth and regular with no fracture or defect. Chondrocytes lined up neatly with four regular structure layering, staining evenly distributed and tidemark being clear and complete (Table 3).

Distinct inflammatory infiltration was observed in synovial tissues of KOA group. Positive staining cells of TRPA1 and TRPV4 were identified in every sample, whose expressions in KOA group were statistically higher than those in both normal and KOA-antagonist groups (Figure 3).
Both TRPA1 and TRPV4 expressions from diseased synovial tissues of KOA group were statistically higher than those of normal group $(p<0.05)$ (Figure 4, Table 4). Both TRPA1 and TRPV4 gene expressions in KOA group were statistically higher than those in normal group $(p<0.05)$ (Figure 5, Table 4).

\section{DISCUSSION}

Our study demonstrated that the rats in KOA group had mechanical hyperalgesia two weeks after modeling, which was manifested by decreased MWTs for over eight weeks. TRPA1

Table 4. Protein and gene expression of transient receptor potential ankyrin 1 and transient receptor potential vanilloid 4 in normal and knee osteoarthritis groups

\begin{tabular}{|c|c|c|c|c|}
\hline \multirow[t]{2}{*}{ Group (n=15) } & TRPA1 (protein) & TRPV41 (protein) & TRPA1 (mRNA) & TRPV4 (mRNA) \\
\hline & Mean \pm SEM & Mean \pm SEM & Mean \pm SEM & Mean \pm SEM \\
\hline Normal & $0.138 \pm 0.071$ & $0.494 \pm 0.074$ & $1.020 \pm 0.237$ & $1.058 \pm 0.089$ \\
\hline Knee osteoarthritis & $0.560 \pm 0.283^{* *}$ & $0.729 \pm 0.130^{* *}$ & $4.418 \pm 1.886^{* *}$ & $3.679 \pm 0.754^{* *}$ \\
\hline
\end{tabular}

SEM: Standard error of the mean; * $\mathrm{p}<0.05,{ }^{* *} \mathrm{p}<0.01$, compared with normal group; TRPA1: Transient receptor potential ankyrin 1 ; TRPV4: Transient receptor potential vanilloid 4; mRNA: Messenger ribonucleic acid. 

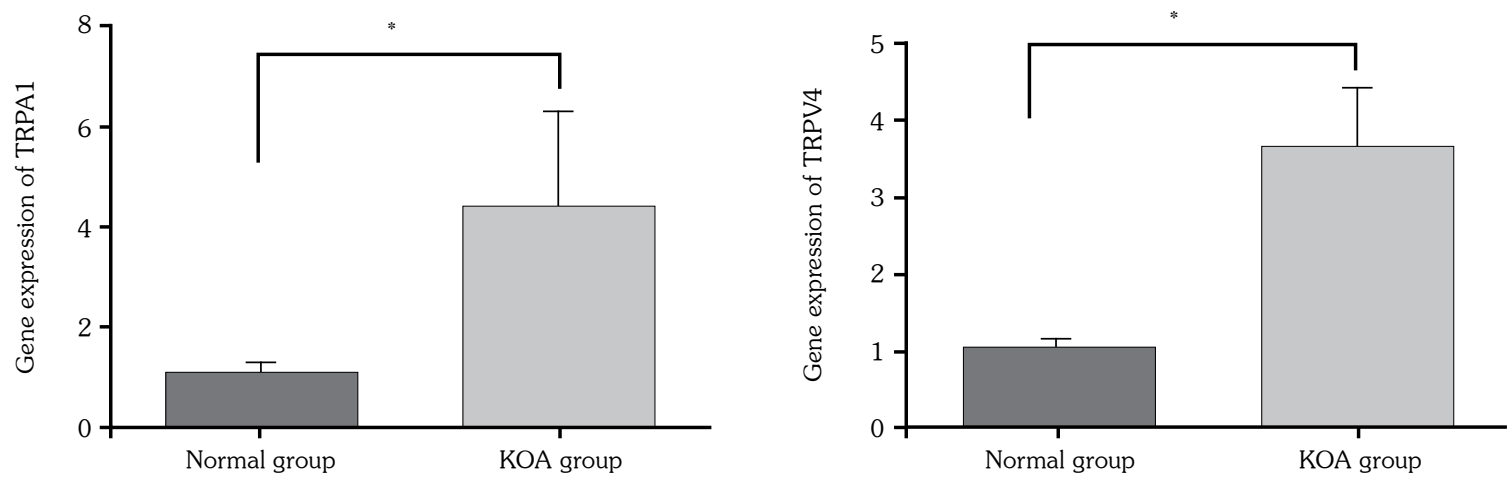

Figure 5. Gene expression of transient receptor potential ankyrin 1 and transient receptor potential vanilloid 4 in synovium tissue of knee joint.

and TRPV4 inhibitors can significantly relieve mechanical hyperalgesia symptoms. MWTs of rats with KOA were lower than those in normal group, and increased after the usage of TRPA1 and TRPV4 inhibitors, which was consistent with behavior indicators. KOA pain displays a typical feature of mechanical hyperalgesia, namely severe persistent pain induced by minor activity, which has not been given full attention in the literature yet. In light of our previous clinical studies and behavioral observations, we designed this animal experimental research to verify our viewpoint.

Transient receptor potential channel has been discovered to be widely distributed in cytomembranes or intracellular organelle membranes of mammals, and works as a primary molecular transducer of mammal body sense system to experience external physical and chemical stimulations (thermal, mechanical, and acid-base). In accordance with relevant literature reports, TRPA1 and TRPV4 work as a sense converter in nociceptive and intense mechanical stimulations induced by osmotic pressure and also play a crucial role in mechanical hyperalgesia. 19,20 $^{2}$ The question whether TRPA1 and TRPV4 participate in KOA pain has attracted considerable attention from researchers.

Our study revealed increased TRPA1 and TRPV4 expressions at synovial tissues of diseased joint in rats with KOA via western blot. A similar result was also obtained after applying immunofluorescence staining. Real time-PCR detected TRPA1 and TRPV4 gene expressions of each group, which were consistent with previous results. After applying selective TRPA1 and
TRPV4 ion channel blockers, as the intracellular $\mathrm{Ca}^{2+}$ concentration was increased, and the epidermal proliferation associated with barrier disruption was reduced, MWTs of rats with KOA were significantly increased, indicating their vital functions in mechanical hyperalgesia occurrence induced by KOA.

All samples were taken from diseased synovial tissues and the secondary dorsal root ganglion in KOA pain conduction was not involved. Some study reports showed that expression changes of TRP channel protein super-family members in dorsal root ganglion can also affect peripheral pain perception, ${ }^{21}$ which will be the focus of our further research. Meanwhile, the time points we selected for determining pain thresholds and measuring TRPA1 and TRPV4 expression levels of each group were one week before modeling, and two, four, six, and eight weeks after modeling, respectively, since two weeks are required for successful modeling and eighth week after modeling was the last time TRPA1 and TRPV4 inhibitors were applied. The close relationship between TRPA1, TRPV4, and the existence of mechanical hyperalgesia in rats with KOA has been verified, yet whether TRPA1 and TRPV4 levels change according to rat mechanical stimulation threshold levels remained unclear. For further research, we are to set up sample collecting time points in accordance with behavior detection to determine TRPA1 and TRPV4 levels and further clarify the relationship between quantity of TRPA1 and TRPV4 and change in tendency of KOA mechanical stimulation threshold levels. 
Limitations of this study include the small number of experimental animal, and we only observed the changes of TRPV4 and TRPA1, the other members of transient receptor potential superfamily would be further investigated in our next studies.

In conclusion, the knee osteoarthritis rats showed mechanical hyperalgesia in the study, and the up-regulating expressions of TRPA 1 and TRPV4 receptors participate in the occurrence of mechanical hyperalgesia induced by knee osteoarthritis.

\section{Declaration of conflicting interests}

The authors declared no conflicts of interest with respect to the authorship and/or publication of this article.

\section{Funding}

The study was supported by NSFC (Natural Science Foundation of China, No. 81573993).

\section{REFERENCES}

1. Johnson VL, Hunter DJ. The epidemiology of osteoarthritis. Best Pract Res Clin Rheumatol 2014;28:5-15.

2. Neogi T. The epidemiology and impact of pain in osteoarthritis. Osteoarthritis Cartilage 2013;21:1145-53.

3. Racine J, Aaron RK. Pathogenesis and epidemiology of osteoarthritis. R I Med J (2013) 2013;96:19-22.

4. Kunimoto $M$. The peripheral mechanism of physiological pain. Brain Nerve 2012;64:1205-14.

5. Filatova ES, Turovskaia EF, Alekseeva LI, Erdes ShF, Nasonov EL. Neurogenic mechanisms of chronic joint pain]. Zh Nevrol Psikhiatr Im S S Korsakova 2013;113:45-9. [Abstract]

6. Rakel B, Vance $\mathrm{C}$, Zimmerman MB, Petsas-Blodgett N, Amendola A, Sluka KA. Mechanical hyperalgesia and reduced quality of life occur in people with mild knee osteoarthritis pain. Clin J Pain 2015;31:315-22.

7. Julius D. TRP channels and pain. Annu Rev Cell Dev Biol 2013;29:355-84.

8. Mickle AD, Shepherd AJ, Mohapatra DP. Sensory TRP channels: the key transducers of nociception and pain. Prog Mol Biol Transl Sci 2015;131:73-118.

9. Zhao P, Lieu T, Barlow N, Sostegni S, Haerteis S, Korbmacher $\mathrm{C}$, et al. Neutrophil Elastase Activates Protease-activated Receptor-2 (PAR2) and Transient Receptor Potential Vanilloid 4 (TRPV4) to Cause Inflammation and Pain. $\mathrm{J}$ Biol Chem 2015;290:13875-87.

10. Chen Y, Kanju P, Fang Q, Lee SH, Parekh PK, Lee W, et al. TRPV4 is necessary for trigeminal irritant pain and functions as a cellular formalin receptor. Pain 2014;155:2662-72.

11. Abdel-Magid AF. TRPV4 Antagonists: Potential treatment for congestive heart failure, bladder dysfunctions, and pain. ACS Med Chem Lett 2013;4:159-60.

12. Pinheiro Fde V, Villarinho JG, Silva CR, Oliveira SM, Pinheiro Kde V, Petri $\mathrm{D}$, et al. The involvement of the TRPA1 receptor in a mouse model of sympathetically maintained neuropathic pain. Eur $\mathrm{J}$ Pharmacol 2015;747:105-13.

13. Hatakeyama Y, Takahashi K, Tominaga M, Kimura H, Ohta T. Polysulfide evokes acute pain through the activation of nociceptive TRPA1 in mouse sensory neurons. Mol Pain 2015;11:24.

14. Jones RC, Xu L, Gebhart GF. The mechanosensitivity of mouse colon afferent fibers and their sensitization by inflammatory mediators require transient receptor potential vanilloid 1 and acid-sensing ion channel 3 . J Neurosci 2005;25:10981-9.

15. Gentry C, Stoakley N, Andersson DA, Bevan S. The roles of iPLA2, TRPM8 and TRPA1 in chemically induced cold hypersensitivity. Mol Pain 2010;6:4.

16. Engler A, Aeschlimann A, Simmen BR, Michel BA, Gay RE, Gay S, et al. Expression of transient receptor potential vanilloid 1 (TRPV1) in synovial fibroblasts from patients with osteoarthritis and rheumatoid arthritis. Biochem Biophys Res Commun 2007;359:884-8.

17. Kelly S, Chapman RJ, Woodhams S, Sagar DR, Turner $\mathrm{J}$, Burston $\mathrm{JJ}$, et al. Increased function of pronociceptive TRPV1 at the level of the joint in a rat model of osteoarthritis pain. Ann Rheum Dis 2015;74:252-9.

18. van der Sluijs JA, Geesink RG, van der Linden AJ, Bulstra SK, Kuyer R, Drukker J. The reliability of the Mankin score for osteoarthritis. J Orthop Res 1992;10:58-61.

19. Mueller-Tribbensee SM, Karna M, Khalil M, Neurath MF, ReehPW,EngelMA.PLoSOne. Differential Contribution of TRPA1, TRPV4 and TRPM8 to Colonic Nociception in Mice 2015;10:0128242.

20. Materazzi S, Fusi C, Benemei S, Pedretti P, Patacchini R, Nilius B, et al. TRPA1 and TRPV4 mediate paclitaxel-induced peripheral neuropathy in mice via a glutathione-sensitive mechanism. Pflugers Arch 2012;463:561-9.

21. Ikeda-Miyagawa $Y$, Kobayashi K, Yamanaka H, Okubo M, Wang S, Dai Y, et al. Peripherally increased artemin is a key regulator of TRPA1/V1 expression in primary afferent neurons. Mol Pain 2015;11:8. 